Preprint typeset in JHEP style - HYPER VERSION

\title{
Black holes in higher spin supergravity
}

\author{
Shouvik Datta, Justin R. David \\ Centre for High Energy Physics, Indian Institute of Science, \\ C.V. Raman Avenue, Bangalore 560012, India. \\ shouvik, justin@cts.iisc.ernet.in
}

\begin{abstract}
We study black hole solutions in Chern-Simons higher spin supergravity based on the superalgebra $s l(3 \mid 2)$. These black hole solutions have a $U(1)$ gauge field and a spin 2 hair in addition to the spin 3 hair. These additional fields correspond to the R-symmetry charges of the supergroup $s l(3 \mid 2)$. Using the relation between the bulk field equations and the Ward identities of a CFT with $\mathcal{N}=2$ super- $\mathcal{W}_{3}$ symmetry, we identify the bulk charges and chemical potentials with those of the boundary CFT. From these identifications we see that a suitable set of variables to study this black hole is in terms of the charges present in three decoupled bosonic sub-algebras of the $\mathcal{N}=2$ super- $\mathcal{W}_{3}$ algebra. The entropy and the partition function of these R-charged black holes are then evaluated in terms of the charges of the bulk theory as well as in terms of its chemical potentials. We then compute the partition function in the dual CFT and find exact agreement with the bulk partition function.
\end{abstract}




\section{Contents}

1. Introduction 1

2. Constructing higher spin black holes 4

2.1 Higher spin Chern-Simons supergravity 1

2.2 On the superalgebra $\operatorname{sl}(3 \mid 2)$

2.3 Black holes in higher spin supergravity 0

3. Bulk equations of motion and Ward identities 8

3.1 Bulk equations of motion 8

3.2 Ward Identities from OPEs 9

3.3 Summary - The AdS/CFT dictionary 11

4. The higher spin black hole 12

4.1 The gauge connections 12

4.2 Black hole holonomy and integrability 13

4.3 Supersymmetry of the higher spin black hole 14

5. Black hole thermodynamics 15

5.1 Entropy in terms of higher spin charges 15

5.2 Entropy and partition function in terms of chemical potentials 17

5.3 Partition function from the dual CFT 19

5.4 Generalizations to higher spin black holes in shs $[\lambda] \quad 20$

6. Conclusions 21

A. OPEs of $\mathcal{N}=2$ super- $\mathcal{W}_{3}$

B. Equivalence of the black hole entropy formulae 24

\section{Introduction}

The study of higher spin theories $(s \geq 2)$ in Anti-de Sitter spaces have been the focus of many recent works (see [1, 2] for comprehensive reviews). These theories offer toy models of AdS/CFT with enhanced symmetry and without the complications of the infinite tower of massive string excitations. Higher spin theories in 3 dimensional 
Anti-de Sitter space are easier to formulate in terms of Chern-Simons theory which renders them more tractable $[4,5,6$, 7]. In three dimensions it is also possible to consistently truncate to a finite set of higher spin fields. There are explicit proposals for the CFT duals of Vasiliev like theories in terms of minimal models for cases with and without supersymmetry [3, 8].

Classical solutions in higher spin theories in 3 dimensional Anti-de Sitter space are easy to construct (see [9] for a recent review). This is because in terms of the Chern-Simons formulation they correspond to a flat connection. Black holes and conical defect solutions constructed in these theories have been used to study the spectrum, nature of singularities with enhanced higher spin symmetry and the holographic renormalization group [10, 11, 12]. Recently the conditions under which a classical solution is supersymmetric in a Chern-Simons higher spin theory was provided in [13, 14]. Supersymmetric classical solutions should correspond to chiral primaries in the boundary CFT and a recent check of this fact has been done in [15].

Conformal field theory in 2 dimensions with enhanced supersymmetry usually admits redefinitions of the currents so that the bosonic sub-algebras mutually commute. Consider the simple case of a CFT with $\mathcal{N}=2$ supersymmetry. The bosonic part of this algebra consists of the stress tensor $T$ and the $U(1)$ current $J$. Redefining the stress tensor as

$$
\hat{T}=T-\frac{3}{2 c} J^{2}
$$

ensures that the new Virasoro algebra commutes with the $U(1)$ current. This redefinition has an important consequence for a BTZ black hole carrying $U(1)$ charge. A bulk theory with $\mathcal{N}=2$ asymptotic algebra is a Chern-Simons theory based on the supergroup $\operatorname{sl}(2 \mid 1)$. The bosonic spectrum of this theory consists of the graviton and a Chern-Simons $U(1)$ gauge field. Consider the BTZ black hole along with the $U(1)$ gauge field. The metric of the black hole is unaffected by the $U(1)$ gauge field. Thus the entropy is independent of the presence of $U(1)$ field. However in the AdS/CFT correspondence, it is $-\hat{T}$ which is identified with the stress tensor of the bulk [16, 17, 18]. This shift is due to the extra energy carried by the gauge field. The Cardy-like formula for the entropy now is given by ${ }^{1}$

$$
S=2 \pi \sqrt{\frac{c}{6}\left(L_{0}-\frac{3}{2 c} J_{0}^{2}\right)}
$$

where $L_{0}$ and $J_{0}$ are the zero modes of the stress tensor and the $U(1)$ current of the CFT. We wish to study this phenomena in the presence of higher spin fields. A natural and consistent framework to introduce an additional $U(1)$ in Vasiliev theories is to study supersymmetric Chern-Simons theories based on the supergroup $\operatorname{sl}(N \mid N-1)$. These theories always contain a $U(1)$ as a R-symmetry. The second

\footnotetext{
${ }^{1}$ See [19] for a derivation.
} 
goal in this paper is to reproduce the partition function of black holes in higher spin supersymmetric theories from the CFT.

Our set up is the Chern-Simons theory based on the supergroup $\operatorname{sl}(3 \mid 2)$ whose bosonic field content include the graviton and a spin 3 field. There is an additional spin 2 field and a $U(1)$ gauge field corresponding to the R-symmetry part of the supergroup $s l(3 \mid 2)$. To discover how the charges in the boundary are affected due to the presence of the $U(1)$ field we use the correspondence of the bulk equations of motion to the Ward identities of the CFT [20]. In this case the Ward identities arise from the semi-classical operator product expansions of the the $\mathcal{N}=2$ super- $\mathcal{W}_{3}$ which is the asymptotic algebra of the $s l(3 \mid 2)$ theory $^{2}$. We see that indeed the stress tensor is shifted precisely as expected. However, there is also a shift of the spin 3 charge which is proportional to the product of the spin 2 R-charge and the $U(1)$ charge. The precise shifts are provided in Table 1 . The bosonic part of the $\mathcal{N}=2$ super- $\mathcal{W}_{3}$ algebra can be written into 3 mutually commuting algebras given by

$$
\mathcal{N}=2 \operatorname{super}-\mathcal{W}_{3} \supset \mathcal{W}^{+} \oplus \mathcal{W}^{-} \oplus u(1)
$$

where $\mathcal{W}^{+}$is a Virasoro algebra and $\mathcal{W}^{-}$is the bosonic $\mathcal{W}_{3}$ algebra [28]. From these shifts found in table 1 , we see that a natural linear combination of the bulk charges are directly identified with the charges in the mutually commuting bosonic algebras. We then construct a black hole solution carrying these charges and evaluate its partition function and entropy. This is done using the integrability conditions satisfied by the holonomy equations. The partition function and the entropy are written both in terms of the charges and the corresponding chemical potentials. We see that the black hole entropy can be written in terms of contributions from the decoupled CFTs. The contribution from the $\mathcal{W}^{+}$part is due to a Virasoro algebra and the entropy from the CFT can be understood in terms of the Cardy formula. The contribution from the $\mathcal{W}^{-}$part to the partition function with higher spin charge has been recently computed in [33. Appealing to this result we show that the entropy of the black hole in the higher spin super-Chern-Simons theory can be exactly reproduced form the CFT. We then discuss the implications of these observations for black holes in theories dual to the Kazama-Suzuki models of [8].

The organization of the paper is as follows: Section 2 reviews the generalities of higher spin super Chern-Simons theory and then details the $\operatorname{sl}(3 \mid 2)$ algebra. We then write down the general form of the Chern-Simons connection which we will be interested in. In Section 3 the correspondence of the bulk equations of motion with the semi-classical Ward identities of the $\mathcal{N}=2$ super- $\mathcal{W}_{3}$ algebra is used to obtain the relationship between the bulk charges and chemical potentials with that of the boundary theory. From these we see that a natural linear combination of bulk charges are directly identified with the charges of the decoupled CFTs given in (1.3). Section

\footnotetext{
${ }^{2}$ Asymptotic algebras of higher spin super Chern-Simons theories were studied in 6,13 .
} 
4 discusses the construction of the higher spin black hole with spin 3 field carrying the spin 2 and $U(1)$ R-charges. It then demonstrates the integrability conditions are satisfied by the holonomy conditions. This implies the existence of consistent thermodynamic description of the black hole. We then evaluate the entropy and the partition function of the black hole solution both in terms of charges and the chemical potentials. We then show that the partition function can be reproduced exactly from the CFT. The conditions for which the black hole is supersymmetric is also analyzed in this section. We then discuss the implications of these observations for black holes in supersymmetric higher spin theories based on the supergroup $\operatorname{shs}[\lambda]$. Section 6 contains the conclusions. Appendix A contains the details of the semiclassical OPEs of the $\mathcal{N}=2$ super $\mathcal{W}_{3}$ algebra. Appendix B demonstrates the equivalence of the entropy written in terms of charges to that written in terms of the chemical potentials.

Note added: While this manuscript was in preparation we received [21] which has some overlap with this paper.

\section{Constructing higher spin black holes}

\subsection{Higher spin Chern-Simons supergravity}

It has been well-known that gravity and its supersymmetric extensions in 3-dimensions can be described by a Chern-Simons theories [22, 23, 24, 25]. Black hole in such gravity theories have also been studied [26]. Higher spin gravity with spins $2,3, \cdots N$ is described by generalizing the gauge group from $S L(2, R) \times S L(2, R)$ to $S L(N, R) \times$ $S L(N, R)$ [ [1, [1, 27]. This can also be phrased in terms of the higher spin algebra $h s[\lambda]$ with $\lambda=-N$. These $h s[\lambda]$ theories have gained considerable interest in the context of its interpretation in terms of holographic minimal models [2]. As in the case for pure gravity one may also look for supersymmetric generalizations of higher spin gravity. The gauge group in such a case is given by $\operatorname{sh} s[\lambda][$ [ $]$. For the case of $\mathcal{N}=2$ higher spin supergravity the gauge group is given by $\operatorname{sl}(N \mid N-1)$. The Chern-Simons action for such a supergravity theory based on a supergroup $\mathcal{G}$ is given by

$$
S=\frac{k}{2 \pi} \int\left[\operatorname{str}\left(A d A+\frac{2}{3} A^{3}\right)-\operatorname{str}\left(\bar{A} d \bar{A}+\frac{2}{3} \bar{A}^{3}\right)\right]
$$

where $A=A_{\mu}^{a} T^{a} d x^{\mu}$, with $T^{a}$ being the generators of the supergroup. The gauge connections are given in terms of the tetrad and vielbein of the background metric as

$$
A=\omega+e \quad \bar{A}=\omega-e
$$

The equation of motion for the action above is the flatness conditions on the gauge connections and is given by

$$
d A+A \wedge A=0 \quad d \bar{A}+\bar{A} \wedge \bar{A}=0
$$


The metric can be obtained from the gauge connection using

$$
g_{\mu \nu}=\frac{1}{\epsilon_{(N \mid N-1)}} \operatorname{str}\left(e_{\mu} e_{\nu}\right)
$$

Here $\epsilon_{(N \mid N-1)}$ is a normalization constant given by $\frac{N(N-1)}{4}$.

\subsection{On the superalgebra $s l(3 \mid 2)$}

We shall be considering black holes in the simplest higher spin supergravity theory. This is based on the algebra $s l(3 \mid 2)$ and has $\mathcal{N}=2$ supersymmetry. This is the semiclassical version $(c \rightarrow \infty)$ of the global part of the $\mathcal{N}=2$ super- $\mathcal{W}_{3}$ algebra [28]. This is a refection of the fact that the asymptotic algebra for this case is that of super- $\mathcal{W}_{3}$ and the dual superconformal field theory has this symmetry.

This algebra has bosonic generators $J, L, V$ and $W$ corresponding to spins 1, 2, 2 and 3 respectively. The algebra formed by just $L$ is the usual $S L(2, R)$ algebra. There are fermionic generators $G^{ \pm}$and $U^{ \pm}$corresponding to spins $3 / 2$ and $5 / 2$ respectively.

We shall list explicit commutation relations for this algebra below.

$$
\begin{aligned}
{[J, J] } & =0, \quad\left[L_{m}, L_{n}\right]=(m-n) L_{m+n}, \\
{\left[V_{m}, V_{n}\right] } & =(m-n)\left(L_{m+n}+\kappa V_{m+n}\right), \\
{\left[W_{m}, W_{n}\right] } & =\frac{1}{4}(m-n)\left(2 m^{2}+2 n^{2}-m n-8\right)\left(L_{m+n}+\frac{\kappa}{5} V_{m+n}\right), \\
{\left[J, L_{n}\right] } & =0, \quad\left[J, V_{n}\right]=0, \quad\left[J, W_{n}\right]=0, \\
{\left[L_{m}, V_{n}\right] } & =(m-n) V_{m+n}, \quad\left[L_{m}, W_{n}\right]=(2 m-n) W_{m+n}, \\
{\left[V_{m}, W_{n}\right] } & =\frac{\kappa}{5}(2 m-n) W_{m+n} .
\end{aligned}
$$

where $\kappa= \pm(5 / 2) i$. Here the subscripts $m, n$ on the generators $L$ run from $-1,0,1$ while the subscripts on the generators $W$ run from $-2,-1,0,1,2$. The commutation relations between bosonic and fermionic generators are given by

$$
\begin{aligned}
{\left[L_{m}, G_{r}^{ \pm}\right] } & =\left(\frac{1}{2} m-r\right) G_{m+n}^{ \pm}, \quad\left[J, G_{r}^{ \pm}\right]= \pm G_{r}^{ \pm}, \\
{\left[L_{m}, U_{r}^{ \pm}\right] } & =\left(\frac{3}{2} m-r\right) U_{m+r}^{ \pm}, \quad\left[J, U_{r}^{ \pm}\right]= \pm U_{r}^{ \pm}, \\
{\left[V_{m}, G_{r}^{ \pm}\right] } & = \pm U_{r+m}^{ \pm}, \quad\left[G_{r}^{ \pm}, W_{m}\right]=\left(2 r-\frac{1}{2} m\right) U_{r+m}^{ \pm}, \\
{\left[V_{m}, U_{r}^{+}\right]=\frac{2}{5} \kappa\left(\frac{3}{2} m-r\right) U_{m+r}^{+}+\frac{1}{4}\left(3 m^{2}-2 m r+r^{2}-\frac{9}{4}\right) G_{m+r}^{+}, } & \\
{\left[V_{m}, U_{r}^{-}\right]=} & -\frac{2}{5} \kappa^{*}\left(\frac{3}{2} m-r\right) U_{m+r}^{-}-\frac{1}{4}\left(3 m^{2}-2 m r+r^{2}-\frac{9}{4}\right) G_{m+r}^{-}, \\
{\left[U_{r}^{+}, W_{m}\right]=\frac{\kappa}{10}\left(2 r^{2}-2 r m+m^{2}-\frac{5}{2}\right) U_{r+m}^{+} } & \quad+\frac{1}{8}\left(4 r^{3}-3 r^{2} m+2 r m^{2}-m^{3}-9 r+\frac{19}{4} m\right) G_{r+m}^{+}, \\
{\left[U_{r}^{-}, W_{m}\right]=\frac{\kappa^{*}}{10}\left(2 r^{2}-2 r m+m^{2}-\frac{5}{2}\right) U_{r+m}^{-} } & \quad+\frac{1}{8}\left(4 r^{3}-3 r^{2} m+2 r m^{2}-m^{3}-9 r+\frac{19}{4} m\right) G_{r+m}^{-} .
\end{aligned}
$$

Here the subscripts $r, s$ on $G^{ \pm}$run from $-1 /, 1 / 2$ while the subscripts on the generators $U^{ \pm}$run from $-3 / 2,-1 / 2,1 / 2,3 / 2$. Finally the anti-commutation rules between 
the fermionic generators are given by

$$
\begin{aligned}
& \left\{G_{r}^{ \pm}, G_{s}^{\mp}\right\}=2 L_{r+s} \pm(r-s) J, \quad\left\{G_{r}^{ \pm}, G_{s}^{ \pm}\right\}=0, \\
& \left\{G_{r}^{ \pm}, U_{s}^{\mp}\right\}=2 W_{r+s} \pm(3 r-s) V_{r+s}, \quad\left\{G_{r}^{ \pm}, U_{s}^{ \pm}\right\}=0, \\
& \left\{U_{r}^{+}, U_{s}^{-}\right\}=-\frac{2}{5} \kappa(r-s) W_{r+s}+\left(3 s^{2}-4 r s+3 r^{2}-\frac{9}{2}\right)\left(\frac{1}{2} L_{r+s}+\frac{\kappa}{5} V_{r+s}\right) \\
& \quad+\frac{1}{4}(r-s)\left(r^{2}+s^{2}-\frac{5}{2}\right) J_{r+s}, \\
& \left\{U_{r}^{ \pm}, U_{s}^{ \pm}\right\}=0 .
\end{aligned}
$$

This algebra has 12 fermionic and bosonic generators each. The bosonic part of the superalgebra is given a direct sum of the subalgebras $\operatorname{sl}(3) \oplus \operatorname{sl}(2) \oplus u(1)$. This can be explicitly seen by defining new spin- 2 generators, $T^{ \pm}$in terms of $L$ and $V$ as follows

$$
T_{m}^{+}=-\frac{1}{3}\left(L_{m}+2 i V_{m}\right) \quad T_{m}^{-}=\frac{1}{3}\left(4 L_{m}+2 i V_{m}\right) .
$$

Substitituting these redefintions in 2.5) we obtain

$$
\left[T_{m}^{+}, T_{n}^{-}\right]=0, \quad\left[T_{m}^{+}, W_{n}\right]=0,
$$

The generators $T_{m}^{+}$obey the $s l(2)$ algebra while the generators $T_{n}^{-}, W_{m}$ obey the commutation relations of the $\operatorname{sl}(3)$ algebra

$$
\begin{aligned}
{\left[T_{m}^{-}, T_{n}^{-}\right] } & =(m-n) T_{m+n}^{-}, \quad\left[T_{m}^{-}, W_{n}\right]=(2 m-n) W_{m+n}, \\
{\left[W_{m}, W_{n}\right] } & =\frac{3}{16}(m-n)\left(2 m^{2}+2 n^{2}-m n-8\right) T_{m+n}^{-} .
\end{aligned}
$$

The $\operatorname{sl}(3)$ algebra above is same as that of as the one given in equation (A.2) of [20] with $\sigma=(3 / 4)^{2}$. For the $\operatorname{sl}(3)$ part we shall be using the same representation of the generators as given in [20] while the for the $\operatorname{sl}(2)$ part the representation in terms of Pauli matrices are used. For the $u(1)$ generator the diagonal matrix $(-2,-2,-2,-3,-3)$ is used as mentioned in Section 61 of [29]. We will choose the gravitational $s l(2)$ generators to be that given by $L_{m}$ and the corresponding supercharges to be $G_{r}^{ \pm}$which form the principal embedding of $\operatorname{ssp}(2 \mid 1)$ in $\operatorname{sl}(3 \mid 2)$. Throughout this paper we will work with this embedding, recently there has been a study of the other embeddings of $\operatorname{osp}(2 \mid 1)$ in $\operatorname{sl}(3 \mid 2)$ in [34 and the renormalization group flows between the various embeddings.

\section{The decomposition of the super- $\mathcal{W}_{3}$ algebra}

The fact that the global generators of the bosonic part of super- $\mathcal{W}_{3}$ decomposes into global $\operatorname{sl}(3) \oplus \operatorname{sl}(2) \oplus u(1)$ also carries over to local generators. It is known that the bosonic subalgebra of the $\mathcal{N}=2$ super- $\mathcal{W}_{3}$ algebra also splits into three mutually commuting pieces as mentioned in [28]. The splitting can be written as

$$
\mathcal{N}=2 \operatorname{super}-\mathcal{W}_{3} \supset \mathcal{W}^{+} \oplus \mathcal{W}^{-} \oplus u(1)
$$


$\mathcal{W}^{+}$contains just a spin-2 generator which is given by $\left(T^{+}+\frac{1}{2 c} J^{2}\right)$ in the large $c$ limit. $\mathcal{W}^{-}$contains a spin-2 generator given by $\left(T^{-}-\frac{2}{c} J^{2}\right)$ and a spin-3 generator which is given by $\left(W-\frac{6}{c} J V\right)$ in the large $c$ limit. The central charges for these subalgebras are

$$
c^{+}=-\frac{1}{3} c, \quad c^{-}=\frac{4}{3} c, \quad c_{J}=1
$$

We have written the field redefinitions and the central charges in the large $c$ limit. The full quantum version of this decomposition and the central charges in each of the commuting sectors are given in [28]. The $\mathcal{W}^{+}$is then a Virasoro algebra with central charge $c^{+}$while $\mathcal{W}^{-}$is a $\mathcal{W}_{3}$ algebra with $c^{-}$as its central charge. This decomposition will play an important role when we evaluate the partition function of the black hole from the dual CFT and show that it precisely agrees with that obtained from the classical solution in the bulk.

\subsection{Black holes in higher spin supergravity}

There has been many recent constructions of classical solutions (black holes and conical defects) in higher spin theories [20, 12, 10]. Similar constructions in higher spin supergravity were studied in 13, 14. They turn out to be interesting in their own right [11, 30, 31, 9] and also from their interpretation in the dual CFT [32, 33, 34]. The thermodynamics of higher spin black holes turn out to be quite interesting and have been recently investigated in [35, 36, 37, 38, 39, 40. Here we shall be interested in constructing black hole solutions and studying their thermodynamics in such higher spin supergravity theories. The trial gauge connections for the higher spin black hole embedded in $s l(3 \mid 2)$ which we shall be considering here are as follows

$$
A=b^{-1} a\left(x^{+}\right) b+b^{-1} d b \quad \bar{A}=b^{-1} \bar{a}\left(x^{+}\right) b+b^{-1} d b
$$

where

$$
\begin{aligned}
& a=\left(L_{1}-\frac{2 \pi}{k} \mathcal{L} L_{-1}-\frac{2 \pi}{k} \mathcal{V} V_{-1}+\frac{\pi}{2 k \sigma} \mathcal{W} W_{-2}+\frac{\pi}{k} \mathcal{J} J_{0}\right) d x^{+} \\
&+\left(\mu W_{2}+w_{1} W_{1}+w_{0} W_{0}+w_{-1} W_{-1}+w_{-2} W_{-2}+\rho V_{1}+v_{0} V_{0}+v_{-1} V_{-1}\right. \\
&\left.\quad+\ell L_{-1}-\gamma J_{0}\right) d x^{-} \\
& \bar{a}=-\left(L_{1}-\frac{2 \pi}{k} \overline{\mathcal{L}} L_{-1}-\frac{2 \pi}{k} \overline{\mathcal{V}} V_{-1}+\frac{\pi}{2 k \sigma} \overline{\mathcal{W}} W_{-2}-\frac{\pi}{k} \mathcal{J} J_{0}\right) d x^{-} \\
&-\left(\bar{\mu} W_{-2}+\bar{w}_{1} W_{1}+\bar{w}_{0} W_{0}+\bar{w}_{-1} W_{-1}+\bar{w}_{2} W_{2}+\bar{\rho} V_{1}+\bar{v}_{0} V_{0}+\bar{v}_{-1} V_{-1}\right. \\
&\left.\quad+\bar{\ell} L_{1}+\gamma J_{0}\right) d x^{+}
\end{aligned}
$$

One is motivated to choose deformations of asymptotically-AdS connections of this form on basis of [0, 20]. The 14 undetermined functions

$$
\left\{\mathcal{J}, \mathcal{L}, \mathcal{V}, \mathcal{W}, \mu, \ell, v_{-1}, v_{0}, \rho, w_{-2}, w_{-1}, w_{0}, w_{1}, w_{2}\right\}
$$


are allowed to depend on $x^{+}(=t+\phi)$ and $x^{-}(=t-\phi)$. It will be seen later that $\gamma, \rho$ and $\mu$ are chemical potentials conjugate to $\mathcal{J}, \mathcal{V}$ and $\mathcal{W}^{3}$.

\section{Bulk equations of motion and Ward identities}

In this section we shall be taking a look at the constraints on $\mathcal{J}, \mathcal{L}, \mathcal{V}$ and $\mathcal{W}$ imposed by the flatness conditions. We then show that we will obtain the same constraints using the Ward identities from the operator product expansions of the $\mathcal{N}=2$ super$\mathcal{W}_{3}$ algebra. In the process we will relate the charges and chemical potentials in the bulk to their counterparts in the boundary. This method was developed in bosonic theories in [20] and applied to supersymmetric theories in [41, 42]. The AdS/CFT dictionary for this case will be obtained. When these charges and currents are used as thermodynamic variables to calculate the entropy and the partition function, the identifications of these quantities with that of the super- $\mathcal{W}_{3} \mathrm{CFT}$ will be important to match the entropy and the partition function obtain from field theory calculations.

\subsection{Bulk equations of motion}

On substituting the connection (2.14) in the Chern-Simons equation of motion $(d a+$ $a \wedge a=0$ ) we get the following considering the coefficients of each of the generators

$$
\begin{aligned}
w_{1}= & -\partial_{+} \mu \\
w_{0}= & +\frac{1}{2} \partial_{+}^{2} \mu-\frac{4 \pi}{k}\left(\mathcal{L}+\frac{i}{2} \mathcal{V}\right) \mu \\
w_{-1}= & -\frac{1}{6} \partial^{3} \mu+\frac{4 \pi}{3 k} \partial_{+}\left(\mathcal{L}+\frac{i}{2} \mathcal{V}\right) \mu+\frac{10 \pi}{3 k}\left(\mathcal{L}+\frac{i}{2} \mathcal{V}\right) \partial_{+} \mu \\
w_{-2}= & \frac{1}{24} \partial_{+}^{4} \mu-\frac{4 \pi}{3 k} \partial_{+}^{2} \mu\left(\mathcal{L}+\frac{i}{2} \mathcal{V}\right)-\frac{7 \pi}{6 k} \partial_{+}\left(\mathcal{L}+\frac{i}{2} \mathcal{V}\right) \partial_{+} \mu-\frac{\pi}{3 k} \partial_{+}^{2}\left(\mathcal{L}+\frac{i}{2} \mathcal{V}\right) \mu \\
& \quad+\frac{4 \pi^{2}}{k^{2}}\left(\mathcal{L}+\frac{i}{2} \mathcal{V}\right)^{2} \mu+\frac{i \pi \mathcal{W} \rho}{4 k \sigma} \\
v_{0}= & -\partial_{+} \rho \\
v_{-1}= & \frac{1}{2} \partial_{+}^{2} \rho+\frac{3 \pi i}{2 k \sigma} \mathcal{W} \mu-\frac{2 \pi}{k}\left(\mathcal{L}+\frac{5 i}{2} \mathcal{V}\right) \rho \\
\ell= & \frac{3 \pi}{k \sigma} \mathcal{W} \mu-\frac{2 \pi}{k} \mathcal{V} \rho
\end{aligned}
$$

\footnotetext{
${ }^{3} \rho$ used in the connection $a$ is the chemical potential conjugate to $\mathcal{V}$ and should not be confused with the radial coordinate.
} 
$\mathcal{L}, \mathcal{V}$ and $\mathcal{W}$ satisfy the following equations

$$
\begin{aligned}
\partial_{-} \mathcal{J}= & \frac{k}{\pi} \partial_{+} \gamma \\
\partial_{-} \mathcal{L}= & -\frac{8}{3} \partial_{+} \mathcal{W} \mu-4 \mathcal{W} \partial_{+} \mu+\partial_{+} \mathcal{V} \rho+2 \mathcal{V} \partial_{+} \rho \\
\partial_{-} \mathcal{V}= & -\frac{k}{4 \pi} \partial_{+}^{3} \rho-\frac{3 i}{4 \sigma} \partial_{+} \mathcal{W} \mu-\frac{9 i}{8 \sigma} \mathcal{W} \partial_{+} \mu+\partial_{+}\left(\mathcal{L}+\frac{5 i}{2} \mathcal{V}\right) \rho+2\left(\mathcal{L}+\frac{5 i}{2} \mathcal{V}\right) \partial_{+} \rho \\
\partial_{+} \mathcal{W}= & \frac{3 i}{2} \mathcal{W} \partial_{+} \rho+\frac{i}{2} \partial_{+} \mathcal{W} \rho+\frac{\sigma k}{12 \pi} \partial_{+}^{5} \mu-\frac{2 \sigma}{3} \partial_{+}^{3}\left(\mathcal{L}+\frac{\kappa}{5} \mathcal{V}\right) \mu-3 \sigma \partial_{+}^{2}\left(\mathcal{L}+\frac{\kappa}{5} \mathcal{V}\right) \partial_{+} \mu \\
& -5 \sigma \partial_{+}\left(\mathcal{L}+\frac{\kappa}{5} \mathcal{V}\right) \partial_{+}^{2} \mu-\frac{10 \sigma}{3}\left(\mathcal{L}+\frac{\kappa}{5} \mathcal{V}\right) \partial_{+}^{3} \mu+\frac{64 \pi \sigma}{3 k}\left(\mathcal{L}+\frac{\kappa}{5} \mathcal{V}\right) \partial_{+}\left(\mathcal{L}+\frac{\kappa}{5} \mathcal{V}\right) \mu \\
& +\frac{64 \pi \sigma}{3 k}\left(\mathcal{L}+\frac{\kappa}{5} \mathcal{V}\right)^{2} \partial_{+} \mu
\end{aligned}
$$

\subsection{Ward Identities from OPEs}

In this section we shall try to obtain the same equations as Ward identities from the OPEs of the $\mathcal{N}=2$ super- $\mathcal{W}_{3}$ algebra [28]. The OPEs are listed in Appendix A.

\section{Spin-1 current, $J$}

The non-vanishing OPEs for operators along with $J(z)$ are that of $J(z) J(w)$ and $J(z) W(w)$. The Ward identity is given by

$$
\partial_{\bar{z}} J(z, \bar{z})=\partial_{\bar{z}} \int d^{2} y(J(z) J(y) \gamma(y)+J(z) W(y) \mu(y))
$$

here by $J(z, \bar{z})$ we mean the expectation value $\langle J\rangle_{\gamma, \rho, \mu}$, weighted with $e^{\frac{1}{2 \pi} \int(\gamma J+\rho V+\mu W)}$. Upon using the OPEs and upon using $\partial_{\bar{z}}\left(\frac{1}{z}\right)=2 \pi \delta^{(2)}(z, \bar{z})$ we get

$$
\partial_{\bar{z}} J=-\frac{c}{3} \partial_{z}\left(\gamma+\frac{6}{c} V \mu\right)
$$

On converting the above equation to the Lorentzian signature $\partial_{-} \rightarrow-\partial_{\bar{z}}, \partial_{+} \rightarrow \partial_{z}$ and using $\frac{k}{2 \pi}=\frac{c}{6}$ we can identify the bulk variables in (3.2) with those from the algebra as

$$
\mathcal{J}=J, \quad \gamma_{\mathrm{bulk}}=\gamma+\frac{6}{c} V \mu
$$

\section{Stress-tensor, $T$}

For this case the non-zero OPEs are that of $T(z) J(y), T(z) V(y)$ and $T(z) W(y)$. The variation is then given as

$$
\partial_{\bar{z}} T(z, \bar{z})=\partial_{\bar{z}} \int d^{2} y(T(z) J(y) \gamma(y)+T(z) V(y) \rho(y)+T(z) W(y) \mu(y))
$$

Substituting the OPE we obtain

$$
\partial_{\bar{z}} T=-\left(2 \partial_{z} W \mu+3 W \partial_{z} \mu+2 V \partial_{z} \rho+\partial_{z} V \rho+J \partial_{z} \gamma\right)
$$


Using (3.6), converting to Lorentzian signature and comparing with (3.3) leads us to

$$
\begin{aligned}
\mathcal{L}=-\left(T-\frac{3}{2 c} J^{2}\right), \quad \mathcal{V}=-V, \quad \mathcal{W} & =\frac{3}{4}\left(W-\frac{6}{c} J V\right), \\
\rho_{\text {bulk }}=\rho+\frac{6}{c} J \mu, \quad \mu_{\text {bulk }} & =\mu
\end{aligned}
$$

along with the ones in (3.8) which remain consistent identifications. Thus, the Ward identities of $J$ and $T$ are sufficient to give all the identifications between the bulk and algebraic variables. We shall now find the Ward identities of $V$ and $W$ and compare them with the constraints from the bulk equations of motion with the identifications given in (3.8) and (3.11). This is performed as an additional consistency check of the identifications.

\section{The other spin-2 current, $V$}

The contributing OPEs in this case are that of $V(z) V(y)$ and $V(z) W(y)$.

$$
\partial_{\bar{z}} V(z, \bar{z})=\partial_{\bar{z}} \int d^{2} y(V(z) V(y) \rho(y)+V(z) W(y) \mu(y))
$$

from which we get

$$
\begin{aligned}
\partial_{\bar{z}} V & =-\left(\frac{c}{12} \partial_{z}^{3} \rho+2\left(T+\kappa V-\frac{3}{2 c} J^{2}\right) \partial_{z} \rho+\rho \partial_{z}\left(T+\kappa V-\frac{3}{2 c} J^{2}\right)\right. \\
& \left.+\frac{2}{c}\left(J \partial_{z}(T+\kappa V)-2 \partial_{z} J(T+\kappa V)\right) \mu+3 \partial_{z} \mu C^{[3]}+2 \partial_{z} C^{[3]} \mu+\partial_{z}^{3}\left(\frac{1}{2} J \mu\right)\right)
\end{aligned}
$$

where $C^{[3]}$ is given in equation (A.10) in the appendix. The identifications in (3.8) and (3.11) can be consistently used in while comparing (3.4) with the Lorentzian version of the above equation and using (3.6) and (3.10).

\section{The spin-3 charge, $W$}

We finally come to the case of the spin-3 charge, $W$. The contributions to the variation arise from $W(z) W(y), W(z) V(y)$ and $W(z) J(y)$ OPEs

$$
\partial_{\bar{z}} W(z, \bar{z})=\partial_{\bar{z}} \int d^{2} y(W(z) W(y) \mu(y) \rho(y)+W(z) V(y) \rho(y)+W(z) J(y) \gamma(y))
$$

Substituting the OPEs we get the following

$$
\begin{aligned}
\partial_{\bar{z}} W(z, \bar{z})= & -\frac{c_{W}}{360} \partial_{z}^{5} \mu-10 B^{[2]} \partial_{z}^{3} \mu-15 \partial_{z} B^{[2]} \partial_{z}^{2} \mu-\left(2 B^{[4]}+9 \partial_{z}^{2} B^{[2]}\right) \partial_{z} \mu \\
& -\left(\partial_{z} B^{[4]}+\partial_{z}^{3} B^{[2]}\right) \mu-C^{[1]} \partial_{z}^{3} \rho-3\left(C^{[3]}-5 \partial_{z} C^{[2]}\right) \partial_{z} \rho-\left(C^{[4]}+\partial_{z} C^{[3]}\right) \rho \\
& +2 V \partial_{z} \gamma
\end{aligned}
$$


Substituting the $B^{[]_{S}}$ and $C^{[]_{S}}$ above from the equation (A.10) and upon using (3.6), (3.10), (3.13) we can see that this equation exactly matches with (3.5) with the identifications given in (3.8) and (3.11). In doing this we require to make an additional identification $c_{W}=\frac{45}{2 \pi} k$. This is consistent with the relation $c_{W}=\frac{15}{2} c$ found in [28 which was arrived at using the Jacobi identities satisfied by the super- $\mathcal{W}_{3}$ algebra. Although the calculations for this specific Ward identity are quite involved, one can explicitly see the matching by comparing each of the coefficients of the derivatives of the chemical potentials $\gamma, \rho$ and $\mu$.

\subsection{Summary - The AdS/CFT dictionary}

The identifications of the charges and chemical potentials in the boundary and the bulk are summarized in the following table

\begin{tabular}{|c|c|c|c|}
\hline & Spin & Bulk & Boundary \\
\hline \hline \multirow{5}{*}{ Charges } & 1 & $\mathcal{J}$ & $J$ \\
& 2 & $\mathcal{L}$ & $-\left(T-\frac{3}{2 c} J^{2}\right)$ \\
& 2 & $\mathcal{V}$ & $-V$ \\
& 3 & $\mathcal{W}$ & $\frac{3}{4}\left(W-\frac{6}{c} J V\right)$ \\
\hline \hline Chemical & 1 & $\gamma$ & $\gamma+\frac{6}{c} V \mu$ \\
potentials & 2 & $\rho$ & $\rho+\frac{6}{c} J \mu$ \\
& 3 & $\mu$ & $\mu$ \\
\hline
\end{tabular}

Table 1 : Relating the charges and currents of the bulk with that of the CFT ${ }^{4}$

These identifications constitute the AdS/CFT dictionary for this higher spin black hole background. It is interesting to note that the charges and chemical potentials shift due to the presence of the R-symmetry part. Note that these identifications satisfied several non-trivial checks. The structure of the $\mathcal{N}=2$ super- $\mathcal{W}_{3}$ OPE's was used to obtain the identifications. The OPE's are of-course constrained by supersymmetry. This is an independent reason for the dual CFT to have the $\mathcal{N}=2$ super- $\mathcal{W}_{3}$ symmetry.

Motivated by the definition (2.8) for the decoupled generators, let us now define the following linear combinations of the charges in the bulk.

$$
\mathcal{T}_{+}=-\frac{1}{3}(\mathcal{L}+2 i \mathcal{V}), \quad \mathcal{T}_{-}=\frac{4}{3}\left(\mathcal{L}+\frac{i}{2} \mathcal{V}\right), \quad \mathcal{W}_{-}=\frac{4}{3} \mathcal{W}
$$

the identifications for the charges become

$$
\mathcal{T}_{+} \rightarrow T_{+}+\frac{1}{2 c} J^{2}, \quad \mathcal{T}_{-} \rightarrow T_{-}-\frac{2}{c} J^{2}, \quad \mathcal{W}_{-} \rightarrow W-\frac{6}{c} J V
$$

Now the bulk charges $\mathcal{T}_{ \pm}, \mathcal{W}_{-}$are identified precisely with the combinations of the boundary currents for which the bosonic part of the $\mathcal{N}=2$ super- $\mathcal{W}_{3}$ algebra decoupled into 3 bosonic sub-algebras as mentioned in subsection 2.2. This shows the

\footnotetext{
${ }^{4}$ Note that in our conventions which is the same as in $20,-\left(T-\frac{3}{2 c} J^{2}\right)$ is positive
} 
natural identification of the decoupled operators in the CFT is in terms of the 'decoupled charges' in the bulk. The combination of the boundary currents which results in the three decoupled bosonic algebra can also be thought of as cosetting out the $U(1)^{5}$. The reason is that, now currents $T_{+}+\frac{1}{2 c} J^{2}$ and $T_{-}-\frac{2}{c} J^{2}$ and $W-\frac{6}{c} J V$ are uncharged with respect to the $U(1)$. Let us again emphasize that this decoupling resulted due to the tight structure of the $\mathcal{N}=2$ super- $\mathcal{W}_{3}$ OPE's. This phenomenon of decoupling is a property of the $\mathcal{N}=2$ super- $\mathcal{W}_{3}$ algebra and is present in the supersymmetric minimal models dual to Chern-Simons theories based on the infinite dimensional supergroup based on $s h s[\lambda]$ as discussed in section 5.4.

\section{The higher spin black hole}

\subsection{The gauge connections}

In this section we write down the connections of the higher spin black hole embedded in the $s l(3 \mid 2)$ theory. If all the charges and chemical potentials are assumed to be

independent of $x_{ \pm}$, then from the equations of motion (3.1) we get the following solution

$$
\begin{aligned}
a=( & \left.L_{1}-\frac{2 \pi}{k} \mathcal{L} L_{-1}-\frac{2 \pi}{k} \mathcal{V} V_{-1}+\frac{\pi}{2 k \sigma} \mathcal{W} W_{-2}+\frac{\pi}{k} \mathcal{J} J_{0}\right) d x^{+} \\
+ & \left(\mu W_{2}-\frac{4 \pi}{k}\left(\mathcal{L}+\frac{i}{2} \mathcal{V}\right) \mu W_{0}+\left(\frac{4 \pi^{2}}{k^{2}}\left(\mathcal{L}+\frac{i}{2} \mathcal{V}\right)^{2} \mu+\frac{i \pi}{4 k \sigma} \mathcal{W} \rho\right) W_{-2}+\rho V_{1}\right. \\
& \left.+\left(\frac{3 \pi i}{2 k \sigma} \mathcal{W} \mu-\frac{2 \pi}{k}\left(\mathcal{L}+\frac{5 i}{2} \mathcal{V}\right) \rho\right) V_{-1}+\left(\frac{3 \pi}{k \sigma} \mathcal{W} \mu-\frac{2 \pi}{k} \mathcal{V} \rho\right) L_{-1}-\frac{\pi}{k} \mathcal{J} J_{0}\right) d x^{-} \\
\bar{a}=- & \left(L_{-1}-\frac{2 \pi}{k} \overline{\mathcal{L}} L_{1}-\frac{2 \pi}{k} \overline{\mathcal{V}} V_{1}+\frac{\pi}{2 k \sigma} \overline{\mathcal{W}} W_{2}-\frac{\pi}{k} \mathcal{J} J_{0}\right) d x^{-} \\
- & \left(\bar{\mu} W_{-2}-\frac{4 \pi}{k}\left(\overline{\mathcal{L}}+\frac{i}{2} \overline{\mathcal{V}}\right) \mu W_{0}+\left(\frac{4 \pi^{2}}{k^{2}}\left(\overline{\mathcal{L}}+\frac{i}{2} \overline{\mathcal{V}}\right)^{2} \mu+\frac{i \pi}{4 k \sigma} \overline{\mathcal{W}} \bar{\rho}\right) W_{2}+\bar{\rho} V_{-1}\right. \\
& \left.\quad+\left(\frac{3 \pi i}{2 k \sigma} \mathcal{W} \mu--\frac{2 \pi}{k}\left(\overline{\mathcal{L}}+\frac{5 i}{2} \overline{\mathcal{V}}\right) \bar{\rho}\right) V_{1}+\left(\frac{3 \pi}{k \sigma} \overline{\mathcal{V}} \bar{\mu}-\frac{2 \pi}{k} \overline{\mathcal{V}} \bar{\rho}\right) L_{1}+\frac{\pi}{k} \mathcal{J} J_{0}\right) d x^{+}
\end{aligned}
$$

The above connection reduces to that of the charged BTZ black hole embedded in the gravitational $s l(2)$ for which the connections are given solely in terms of $L$ and $J$ generators. This can be seen by setting $\mu=\bar{\mu}=\mathcal{W}=\overline{\mathcal{W}}=\rho=\bar{\rho}=\mathcal{V}=\overline{\mathcal{V}}=0$. Note that this black hole is however not continuously connected to the higher spin black hole of [20]. The reason is that even though if one sets $\rho=\bar{\rho}=\mathcal{V}=\overline{\mathcal{V}}=0$, the coefficient of $V_{-1}$ does not vanish.

\footnotetext{
${ }^{5}$ This point was mentioned to us by Matthias Gaberdiel.
} 


\subsection{Black hole holonomy and integrability}

We shall now investigate the holonomy for the higher spin black hole constructed. The holonomy is a gauge invariant and meaningful quantity in Chern-Simons theory. We shall also see that this observable will play a role in determining the entropy for the black hole. We shall be determining the holonomies along the thermal circle and then demand their eigenvalues to be the same as the one for the BTZ black hole.

The holonomy around the Euclidean time circle $(z, \bar{z}) \rightarrow(z+2 \pi \tau, \bar{z}+2 \pi \bar{\tau})$ is

$$
H=b^{-1} e^{\omega} b, \quad \bar{H}=b e^{\bar{\omega}} b^{-1}
$$

where

$$
\omega=2 \pi\left(\tau a_{+}-\bar{\tau} a_{-}\right), \quad \bar{\omega}=2 \pi\left(\tau \bar{a}_{+}-\bar{\tau} \bar{a}_{-}\right)
$$

For the gauge connections embedded in the $\operatorname{sl}(3 \mid 2)$ theory, $\omega$ has the block diagonal form $\operatorname{sl}(3) \oplus \operatorname{sl}(2)$. The eigenvalues of the holonomy matrix for the BTZ black hole is $(-2 \pi i, 0,2 \pi i)$ and $(-\pi i, \pi i)$.

On defining

$$
\alpha_{1}=\bar{\tau} \gamma, \quad \alpha_{2}=\bar{\tau} \rho, \quad \alpha_{3}=\bar{\tau} \mu
$$

the $\operatorname{sl}(2)$ part of the holonomy for the gauge connection given in (4.1) is

$$
\omega_{s l(2)}=\left(\begin{array}{ll}
-\frac{6 \pi\left(\alpha_{1} k+\pi \mathcal{J} \tau\right)}{k} & \frac{4 \pi^{2}(\mathcal{L}+2 i \mathcal{V})\left(\tau-2 i \alpha_{2}\right)}{k} \\
2 \pi\left(\tau-2 i \alpha_{2}\right) & -\frac{6 \pi\left(\alpha_{1} k+\pi \mathcal{J} \tau\right)}{k}
\end{array}\right)
$$

On finding the eigenvalues of the above matrix and equating them $(i \pi,-i \pi){\text { we } \operatorname{get}^{6}}^{6}$

$$
\alpha_{1} k+\pi \mathcal{J} \tau=0
$$

Then (4.6) becomes

$$
\omega_{s l(2)}=\left(\begin{array}{cc}
0 & \frac{4 \pi^{2}\left(\tau-2 i \alpha_{2}\right)(\mathcal{L}+2 i \mathcal{V})}{k} \\
2 \pi\left(\tau-2 i \alpha_{2}\right) & 0
\end{array}\right)
$$

while the $\operatorname{sl}(3)$ part is given by

$\omega_{s l(3)}=\left(\begin{array}{ccc}\frac{16 \pi^{2}\left(\mathcal{L}+\frac{i \mathcal{V}}{2}\right) \sqrt{-\sigma} \alpha_{3}}{3 k} \frac{\pi^{2}\left(8\left(\mathcal{L}+\frac{i \mathcal{V}}{2}\right) \sigma\left(\tau-\frac{i \alpha_{2}}{2}\right)+9 \mathcal{W} \alpha_{3}\right)}{k \sigma} & \frac{4 \pi^{2}\left(16 \pi\left(\mathcal{L}+\frac{i \mathcal{V}}{2}\right)^{2} \sigma \alpha_{3}-2 k \mathcal{W}\left(\tau-\frac{i \alpha_{2}}{2}\right)\right)}{k^{2} \sqrt{-\sigma}} \\ 2 \pi\left(\tau-\frac{i \alpha_{2}}{2}\right) & -\frac{32 \pi^{2}\left(\mathcal{L}+\frac{i \mathcal{V}}{2}\right) \sqrt{-\sigma} \alpha_{3}}{3 k} & \frac{\pi^{2}\left(8\left(\mathcal{L}+\frac{i \mathcal{V}}{2}\right) \sigma\left(\tau-\frac{i \alpha_{2}}{2}\right)+9 \mathcal{W} \alpha_{3}\right)}{k \sigma} \\ -4 \pi \sqrt{-\sigma} \alpha_{3} & 2 \pi\left(\tau-\frac{i \alpha_{2}}{2}\right) & \frac{16 \pi^{2}\left(\mathcal{L}+\frac{i \mathcal{V}}{2}\right) \sqrt{-\sigma} \alpha_{3}}{3 k}\end{array}\right)$

\footnotetext{
${ }^{6}$ There exist two possibilities here regarding whether to equate an eigenvalue to $i \pi$ or $-i \pi$. However, the condition on $\mathcal{J}$ and $\alpha_{1}$ we write down is common to both the cases.
} 
The combinations appearing above motivates us to define a new set of variables as follows

$$
\begin{array}{lll}
\eta_{+}=\tau-2 i \alpha_{2}, & \mathcal{T}_{+}=-\frac{1}{3}(\mathcal{L}+2 i \mathcal{V}), & k_{+}=-\frac{1}{3} k \\
\eta_{-}=\tau-\frac{i}{2} \alpha_{2}, & \mathcal{T}_{-}=\frac{4}{3}\left(\mathcal{L}+\frac{i}{2} \mathcal{V}\right), & k_{-}=\frac{4}{3} k \\
& \mathcal{W}_{-}=\frac{4}{3} \mathcal{W} &
\end{array}
$$

The combinations for $\mathcal{T}_{ \pm}$and $\mathcal{W}_{-}$were mentioned previously in (3.16). This is due to the fact that the bosonic part of $\operatorname{sl}(3 \mid 2)$ splits as $s l(3) \oplus \operatorname{sl}(2) \oplus u(1)$.

We shall now demand that the eigenvalues of holonomy should equal that of the BTZ black hole. An equivalent way of saying this is

$$
\begin{aligned}
\operatorname{tr}\left(\omega_{s l(2)}^{2}\right) & =-2 \pi^{2}, \\
\operatorname{tr}\left(\omega_{s l(3)}^{2}\right) & =-8 \pi^{2}, \\
\operatorname{det}\left(\omega_{s l(3)}\right) & =0 .
\end{aligned}
$$

The first condition gives

$$
\eta_{+}=\frac{i k_{+}}{2} \frac{1}{\sqrt{2 \pi k_{+} \mathcal{T}_{+}}}
$$

the second condition for $\alpha_{3}=0=\mathcal{W}_{\text {- gives }}$

$$
\left.\eta_{-}\right|_{\alpha=0=\mathcal{W}_{-}}=\frac{i k_{-}}{2} \frac{1}{\sqrt{2 \pi k_{-} \mathcal{T}_{-}}}
$$

for non-zero $\alpha_{3}$ and $\mathcal{W}$ we have

$$
256 \pi^{2} \sigma \alpha_{3}^{2} \mathcal{T}_{-}^{2}-24 \pi k_{-} \eta_{-}^{2} \mathcal{T}_{-}-72 \pi k_{-} \eta_{-} \alpha_{3} \mathcal{W}_{-}-3 k_{-}^{2}=0
$$

and from the determinant condition we get

$$
\begin{aligned}
2048 \pi^{2} \sigma^{2} \alpha_{3}^{3} \mathcal{T}_{-}^{3}+576 \pi \sigma k_{-} \eta_{-}^{2} \alpha_{3} \mathcal{T}_{-}^{2} & +864 \pi \sigma k_{-} \alpha_{3}^{2} \eta_{-} \mathcal{W}_{-} \mathcal{T}_{-} \\
& +864 \pi \sigma k_{-} \alpha_{3}^{3} \mathcal{W}_{-}^{2}-27 k_{-}^{2} \eta_{-}^{3} \mathcal{W}_{-}=0
\end{aligned}
$$

These are the same conditions as (5.14) of [20] with $\mathcal{L} \rightarrow \mathcal{T}_{-}, \tau \rightarrow \eta_{-}$and $\alpha \rightarrow \alpha_{3}$. The integrability conditions are then given by

$$
\frac{\partial \mathcal{W}_{-}}{\partial \eta_{-}}=\frac{\partial \mathcal{T}_{-}}{\partial \alpha_{3}}
$$

\subsection{Supersymmetry of the higher spin black hole}

We now analyse the supersymmetry of this higher spin black hole. In [14] it was shown that the Killing spinor can be written in terms of products of background holonomies and odd-roots of the superalgebra. For calculational simplicity we shall restrict ourselves to the case $\mathcal{W}=0$. 
The matrix $a_{\phi}$ is given in terms of the Cartan matrices $H_{i}$ of the superalgebra [29] as

$$
\begin{gathered}
S a_{\phi} S^{-1}=\left(-\frac{4 \pi i \mu \mathcal{T}_{-}}{k}+i(\rho+2 i) \sqrt{\frac{2 \pi \mathcal{T}_{-}}{k}}\right) H_{1}+\left(\frac{4 \pi i \mu \mathcal{T}_{-}}{k}+i(\rho+2 i) \sqrt{\frac{2 \pi \mathcal{T}_{-}}{k}}\right) H_{2} \\
+(2 \rho+i) \frac{2 \pi \mathcal{T}_{+}}{k} H_{\overline{4}}+\left(\frac{\pi \mathcal{J}}{k}+\gamma\right) J
\end{gathered}
$$

where $\mathcal{L}_{1,2}$ are defined in (4.10). Using the subalgebra for $s l(3 \mid 2)$ one can derive the supersymmetric conditions using

$$
\lambda^{r} \alpha_{i}^{r} \pm\left(\frac{\pi \mathcal{J}}{k}+\gamma\right)=i n_{i} \quad n_{i} \in \mathbb{Z}
$$

where $r$ is the index for the Cartan matrices and $i$ is the fermionic direction which one chooses. $\alpha_{i}^{r} \mathrm{~s}$ are the odd-roots of the superalgebra. The commutation relations with the Cartan matrices with the fermionic generators are

$$
\begin{gathered}
{\left[H_{1}, E_{\bar{\iota}, 1}\right]=E_{\bar{\iota}, 1}, \quad\left[H_{1}, E_{\bar{\iota}, 2}\right]=-E_{\bar{\iota}, 2}, \quad\left[H_{1}, E_{\bar{\iota}, 3}\right]=0} \\
{\left[H_{2}, E_{\bar{\imath}, 1}\right]=0, \quad\left[H_{2}, E_{\bar{\iota}, 2}\right]=E_{\bar{\iota}, 2}, \quad\left[H_{3}, E_{\bar{\iota}, 3}\right]=-E_{\bar{\iota}, 3}} \\
{\left[H_{\overline{4}}, E_{\overline{4} i}\right]=-E_{\overline{4} i}, \quad\left[H_{\overline{4}}, E_{\overline{5} i}\right]=-E_{\overline{5} i}}
\end{gathered}
$$

The supersymmetric condition(s) for the fermionic direction $\bar{\iota}=\overline{4}, j=2$ using (4.20) then turn out to be

$$
\begin{aligned}
-\left(-\frac{4 \pi i \mu \mathcal{T}_{-}}{k}+i(\rho+2 i) \sqrt{\frac{2 \pi \mathcal{T}_{-}}{k}}\right)+ & \left(\frac{4 \pi i \mu \mathcal{T}_{-}}{k}+i(\rho+2 i) \sqrt{\frac{2 \pi \mathcal{T}_{-}}{k}}\right) \\
& -(2 \rho+i) \frac{2 \pi \mathcal{T}_{+}}{k}+\left(\frac{\pi \mathcal{J}}{k}+\gamma\right)=i n
\end{aligned}
$$

One can similarly find 5 more conditions for the other fermionic directions.

\section{Black hole thermodynamics}

\subsection{Entropy in terms of higher spin charges}

The integrability conditions (4.18) can be used as Maxwell relations for studying thermodynamics of black holes in higher spin supergravity. We are thus led to define the conjugate variable relations as follows

$$
\eta_{-}=\frac{i}{4 \pi^{2}} \frac{\partial S_{s l(3)}}{\partial \mathcal{T}_{-}}, \quad \alpha_{3}=\frac{i}{4 \pi^{2}} \frac{\partial S_{s l(3)}}{\partial \mathcal{W}_{-}}
$$

and also

$$
\eta_{+}=\frac{i}{4 \pi^{2}} \frac{\partial S_{s l(2)}}{\partial \mathcal{T}_{+}}
$$


The total entropy from the connection $A$ is given by

$$
S=S_{s l(2)}\left(\mathcal{T}_{+}\right)+S_{s l(3)}\left(\mathcal{T}_{-}, \mathcal{W}_{-}\right)
$$

This can be understood as follows. The gauge connection we are considering has a block diagonal form. The Chern-Simons action can then be written as a sum of two contributions from each of these blocks. Since the partition function involves $e^{-I}$, the entropy can be written as the sum above.

Using (4.14) in (5.2) we get

$$
S_{s l(2)}=2 \pi \sqrt{2 \pi k_{+} \mathcal{T}_{+}}
$$

The entropy contribution from the $s l(3)$ part can calculated as follows. We substitute (5.1) in the trace condition (4.16) and then solve the resulting differential equation. On basis of (4.15) and [20] one can choose the following anzatz

$$
S_{s l(3)}=2 \pi \sqrt{2 \pi k_{-} \mathcal{T}_{-}} f(y) \quad \text { where, } y=-\frac{27 k_{-} \mathcal{W}_{-}^{2}}{64 \sigma \pi \mathcal{T}_{-}^{3}}
$$

Substituting this in the differential equation we get

$$
36 y(2-y)\left(f^{\prime}\right)^{2}+f^{2}-1=0
$$

which has the solution

$$
f(y)=\cos \left(\frac{1}{6} \arctan \left(\frac{\sqrt{y(2-y)}}{1-y}\right)\right)
$$

Thus the final result for the entropy is

$$
\begin{aligned}
& S=2 \pi( \sqrt{2 \pi k_{+} \mathcal{T}_{+}}+\sqrt{2 \pi k_{-} \mathcal{T}_{-}} f\left(-\frac{27 k_{-} \mathcal{W}_{-}^{2}}{64 \sigma \pi \mathcal{T}_{-}^{3}}\right) \\
&\left.+\sqrt{2 \pi k_{+} \overline{\mathcal{T}}_{+}}+\sqrt{2 \pi k_{-} \overline{\mathcal{T}}_{-}} f\left(-\frac{27 k_{-} \overline{\mathcal{W}}_{-}^{2}}{64 \sigma \pi \overline{\mathcal{T}}_{-}^{3}}\right)\right)
\end{aligned}
$$

This has the series expansion

$$
\begin{aligned}
S=2 \pi & \sqrt{2 \pi k_{+} \mathcal{T}_{+}}+2 \pi \sqrt{2 \pi k_{-} \mathcal{T}_{-}}\left(1+\frac{3 k_{-}}{256 \pi \sigma} \frac{\mathcal{W}_{-}^{2}}{\mathcal{T}_{-}^{3}}-\frac{105 k_{-}^{2}}{131072 \pi^{2} \sigma^{2}} \frac{\mathcal{W}_{-}^{4}}{\mathcal{T}_{-}^{6}}+\cdots\right) \\
& + \text { barred part }
\end{aligned}
$$

Note that setting $\mathcal{W}_{-}=\mathcal{V}=0$, the entropy reduces to

$$
S=2 \pi \sqrt{2 \pi k \mathcal{L}} .
$$

Here we have used the definitions in (4.10) to rewrite the expression in terms of $k$ and $\mathcal{L}$. Thus we have obtained the expected answer of the entropy of the BTZ black hole 
embedded in the gravitational $s l(2)$ (constructed out of $L_{m}\left(=T_{m}^{+}+T_{m}^{-}\right)$generators). This serves as an additional check of the fact that the the entropy is the sum of the contributions from the $s l(2)$ and the $s l(3)$ part of the Chern-Simons action.

Recently there has been a discussion of entropy of higher spin black holes using the canonical definition of energy in [39, 40] called the 'canonical formalism'. This method differs from that used by [20, 12] which relies on the existence of the partition function and the compatibility with the first law called the 'holomorphic formalism'. The difference arises due to choice of boundary terms. As mentioned in [40], the method [20, 12] is more suited from the CFT point of view due to the existence of the partition function. The CFT computations agree precisely with that of the holomorphic formalism [33]. Our goal in this paper is also to explain the bulk partition function using the CFT. Therefore we have generalized the holomorphic formalism of [20] to obtain the entropy of the supersymmetric black hole.

\subsection{Entropy and partition function in terms of chemical potentials}

We wish to write the formula we just obtained for the entropy of the black hole (5.8) in terms of the chemical potentials. This shall enable us to match the answer from conformal field theory. The partition function of the higher spin black hole embedded in $\operatorname{sl}(3 \mid 2) \oplus \operatorname{sl}(3 \mid 2)$ is $^{7}$

$$
Z_{\text {gravity }}=\left\langle e^{2 \pi i\left(\alpha_{1} \mathcal{J}+\eta_{+} \mathcal{T}_{+}+\eta_{-} \mathcal{T}_{-}+\alpha_{3} \mathcal{W}_{-}\right)}\right\rangle_{\mathrm{BH}}
$$

This can be written in terms of the conventional charges and chemical potentials as

$$
Z_{\text {gravity }}=\left\langle e^{2 \pi i\left(\tau \mathcal{L}+\alpha_{1} \mathcal{J}-\alpha_{2} \mathcal{V}+\frac{4}{3} \alpha_{3} \mathcal{W}\right)}\right\rangle_{\mathrm{BH}}
$$

here 'BH' indicates that the quantity is evaluated for the higher spin black hole background. We thus have the following conjugate variable relations

$$
\begin{array}{rlrl}
\alpha_{1}=\frac{i}{4 \pi^{2}} \frac{\partial S}{\partial \mathcal{J}}, & & \frac{\partial \log Z}{\partial \alpha_{1}}=4 \pi^{2} i \mathcal{J} \\
\eta_{+}=\frac{i}{4 \pi^{2}} \frac{\partial S}{\partial \mathcal{T}_{+}}, & \frac{\partial \log Z}{\partial \eta_{+}}=4 \pi^{2} i \mathcal{T}_{+} \\
\eta_{-}=\frac{i}{4 \pi^{2}} \frac{\partial S}{\partial \mathcal{T}_{-}}, & \frac{\partial \log Z}{\partial \eta_{-}}=4 \pi^{2} i \mathcal{T}_{-} \\
\alpha_{3}=\frac{i}{4 \pi^{2}} \frac{\partial S}{\partial \mathcal{W}}, & \frac{\partial \log Z}{\partial \alpha_{3}}=4 \pi^{2} i \mathcal{W}
\end{array}
$$

In [32] it was shown that the partition function for the hs $[\lambda]$ black hole could be written in terms of the chemical potentials. The approach taken was to assume a power series solution for $\mathcal{L}, \mathcal{W}$ and other higher spin charges, plug them into

\footnotetext{
${ }^{7}$ We are considering just one $\operatorname{sl}(3 \mid 2)$ or the holomorphic part.
} 
the holonomy conditions and solve for the unknown coefficients in the power series expansions. Finally upon integrating the solutions for the charges, one can obtain the partition function. On performing a similar analysis as the above we obtain (for the holomorphic part with terms up to $\left.O\left(\alpha_{3}^{4}\right)\right)$

$$
\begin{aligned}
\mathcal{T}_{+} & =-\frac{c^{+}}{48 \pi \eta_{+}^{2}} \\
\mathcal{T}_{-} & =-\frac{c^{-}}{48 \pi \eta_{-}^{2}}-\frac{5 c^{-} \sigma}{36 \pi \eta_{-}^{6}} \alpha_{3}^{2}-\frac{10 c^{-} \sigma^{2}}{9 \pi \eta_{-}^{10}} \alpha_{3}^{4}+\cdots \\
\mathcal{W}_{-} & =\frac{c^{-} \sigma}{18 \pi \eta_{-}^{5}} \alpha_{3}+\frac{40 c^{-} \sigma^{2}}{81 \pi \eta_{-}^{9}} \alpha_{3}^{3}+\cdots
\end{aligned}
$$

The holonomy condition imposed the following relation on $\mathcal{J}$ and $\alpha_{1}$.

$$
\alpha_{1} k+\pi \mathcal{J} \tau=0
$$

from which we get

$$
\alpha_{1}=-\frac{3 \tau \mathcal{J}}{c}, \quad \mathcal{J}=-\frac{c \alpha_{1}}{3 \tau}
$$

To study the thermodynamics, we have the following relations

$$
\tau=\frac{i}{4 \pi^{2}}\left(\frac{\partial S}{\partial \mathcal{L}}\right)_{\mathcal{L}, \mathcal{V}, \mathcal{W}}, \quad \alpha_{1}=\frac{i}{4 \pi^{2}}\left(\frac{\partial S}{\partial \mathcal{J}}\right)_{\mathcal{J}, \mathcal{V}, \mathcal{W}} .
$$

Using it in the second equation of (5.21) we get

$$
\mathcal{J}=-\frac{c}{3}\left(\frac{\partial S}{\partial \mathcal{J}}\right)_{\mathcal{L}, \mathcal{V}, \mathcal{W}}\left(\frac{\partial S}{\partial \mathcal{L}}\right)_{\mathcal{J}, \mathcal{V}, \mathcal{W}}^{-1}=\frac{c}{3}\left(\frac{\partial \mathcal{L}}{\partial \mathcal{J}}\right)_{S, \mathcal{V}, \mathcal{W}}=0
$$

This implies that the $u(1)$ charge is forced to be zero by the holonomy condition and it does not contribute to the partition function. This conclusion can also be reached from the second relation in (5.22). Since, the entropy is independent of $\mathcal{J}$ we obtain $\alpha_{1}=0$. From the holonomy conditions this then implies $\mathcal{J}=0$. However it was important to keep track of the $\mathcal{J}$ to obtain the redefinitions given in Table 1.

On integrating (5.17), (5.18) and (5.19) we obtain the partition function to be

$$
\log Z_{\text {gravity }}\left(\eta_{+}, \eta_{-}, \alpha_{3}\right)=\frac{\pi i c^{+}}{12 \eta_{+}}+\frac{\pi i c^{-}}{12 \eta_{-}}\left(1+\frac{4 \sigma}{3} \frac{\alpha_{3}^{2}}{\eta_{-}^{4}}+\frac{160 \sigma^{2}}{27} \frac{\alpha_{3}^{4}}{\eta_{-}^{8}}+\cdots\right)
$$

where we have used $k_{ \pm}=c_{ \pm} / 6$. On exponentiating the above, we get

$$
Z_{\text {gravity }}=q^{-c / 24}\left(1+\frac{2 \pi i c^{-}}{\eta_{-}}\left(\frac{\sigma}{18} \frac{\alpha_{3}^{2}}{\eta_{-}^{4}}-\frac{20 \sigma^{2}}{81} \frac{\alpha_{3}^{4}}{\eta_{-}^{8}}+\cdots\right)\right)
$$

Upon using $S=\log Z\left(\eta_{+}, \eta_{-}, \alpha_{3}\right)-4 \pi^{2} i\left(\eta_{+} \mathcal{T}_{+}+\eta_{-} \mathcal{T}_{-}+\alpha_{3} \mathcal{W}\right)$, the entropy is

$$
S\left(\eta_{+}, \eta_{-}, \alpha_{3}\right)=\frac{i \pi c^{+}}{6 \eta_{+}}+\frac{i \pi c^{-}}{6 \eta_{-}}\left(1+\frac{8 \sigma}{3} \frac{\alpha_{3}^{2}}{\eta_{-}^{4}}+\frac{160 \sigma^{2}}{9} \frac{\alpha_{3}^{4}}{\eta_{-}^{4}}+\cdots\right)
$$


The equivalence of the above formula with the one in terms of the charges (5.9) is shown in Appendix B.

Using (4.10) the partition function (5.26) reads as (with $\sigma=9 / 16$ )

$$
\log Z_{\text {gravity }}=\frac{\pi i c}{12 \tau}\left(1+\frac{\alpha_{2}^{2}}{\tau^{2}}+\frac{\alpha_{3}^{2}}{\tau^{4}}+\cdots\right)
$$

\subsection{Partition function from the dual CFT}

We shall now calculate the partition function from the dual CFT. As was noted earlier the bosonic part of $\mathcal{N}=2$ super- $\mathcal{W}_{3}$ algebra splits as $\mathcal{W}_{+} \oplus \mathcal{W}_{-} \oplus u(1)$. The expression for the partition function is then given by

$$
Z_{\mathrm{CFT}}=\operatorname{tr}_{\mathcal{H}}\left[e^{2 \pi i\left(\hat{\eta}_{+}\left(T_{0}^{+}+\frac{1}{2 c} J_{0}^{2}-\frac{c^{+}}{24}\right)+\hat{\eta}_{-}\left(T_{0}^{-}-\frac{2}{c} J_{0}^{2}-\frac{c^{-}}{24}\right)+\hat{\alpha}_{3}\left(W_{0}-\frac{6}{c}(J V)_{0}\right)+\hat{\alpha}_{1} J_{0}\right)}\right]
$$

Note that this is the natural CFT partition function one would write down since the $\mathcal{N}=2$ super- $\mathcal{W}_{3}$ algebra splits as $\mathcal{W}_{+} \oplus \mathcal{W}_{-} \oplus u(1)$. The partition function simply evaluates the number of states weighted with the charges of decoupled bosonic currents. The chemical potentials appearing here are those in the CFT and are not the same as those in gravity. We would now like to write the CFT partition function in terms of the chemical potentials appearing the bulk. The relation between the two sets of chemical potential is given in Table 1. Using the relations in Table 1 and similar definitions as given in (4.5) and (4.10) for the chemical potentials in the CFT we can obtain the the following identity

$$
\hat{\eta}_{+}\left(T_{0}^{+}+\frac{1}{2 c} J_{0}^{2}\right)+\hat{\eta}_{-}\left(T_{0}^{-}-\frac{2}{c} J_{0}^{2}\right)+\hat{\alpha}_{1} J_{0}=\eta_{+}\left(T_{0}^{+}+\frac{1}{2 c} J_{0}^{2}\right)+\eta_{-}\left(T_{0}^{-}-\frac{2}{c} J_{0}^{2}\right)+\alpha_{1} J_{0}
$$

This identity allows the rewriting of the CFT partition function in terms of the chemical potentials of the bulk. Note that this non-trivial identity was arrived at by using the relations given in Table 1 which resulted from the structure of the $\mathcal{N}=2$ super $\mathcal{W}_{3}$ algebra. Here again supersymmetry played a role in this crucial simplification. The partition function then becomes

$$
Z_{\mathrm{CFT}}=q^{-c / 24} \operatorname{tr}_{\mathcal{H}}\left[e^{2 \pi i\left(\eta_{+}\left(T_{0}^{+}+\frac{1}{2 c} J_{0}^{2}\right)+\eta_{-}\left(T_{0}^{-}-\frac{2}{c} J_{0}^{2}\right)+\alpha_{3}\left(W_{0}-\frac{6}{c}(J V)_{0}\right)+\alpha_{1} J_{0}\right)}\right]
$$

Since the $\mathcal{W}_{+}, \mathcal{W}_{-}$and $u(1)$ are mutually commuting subalgebras, we have

$$
Z_{\mathrm{CFT}}=q^{-c / 24} \operatorname{tr}_{\mathcal{H}}\left[e^{2 \pi i \eta_{+}\left(T_{0}^{+}+\frac{1}{2 c} J_{0}^{2}\right)} e^{2 \pi i\left(\eta_{-}\left(T_{0}^{-}-\frac{2}{c} J_{0}^{2}\right)+\alpha_{3}\left(W_{0}-\frac{6}{c}(J V)_{0}\right)\right)} e^{2 \pi i \alpha_{1} J_{0}}\right]
$$

and also the full Hilbert space of states in the CFT factorizes as

$$
\mathcal{H}=\mathcal{H}_{+} \oplus \mathcal{H}_{-} \oplus \mathcal{H}_{u(1)}
$$


The $u(1)$ part does not contribute to the partition function for our case since we are restricting ourselves to the zero charge sector as seen in (5.23). The partition function can then be written as contributions from the Virasoro $\left(\mathcal{W}_{+}\right)$and the $\mathcal{W}_{3}$ $\left(\mathcal{W}_{-}\right)$parts.

$$
Z_{\mathrm{CFT}}=q^{-c / 24} \operatorname{tr}_{\mathcal{H}_{+}}\left[e^{2 \pi i \eta_{+}\left(T_{0}^{+}+\frac{1}{2 c} J_{0}^{2}\right)}\right] \operatorname{tr}_{\mathcal{H}_{-}}\left[e^{2 \pi i\left(\eta_{-}\left(T_{0}^{-}-\frac{2}{c} J_{0}^{2}\right)+\alpha_{3}\left(W_{0}-\frac{6}{c} J_{0} V_{0}\right)\right)}\right]
$$

We shall calculate the partition function at high temperature regime $1 / \tau \rightarrow 0$. The leading term for the Virasoro part is 1. Plugging in the answer calculated for the $\mathcal{W}_{3}$ part from [33] (with $\lambda=3, c \rightarrow c^{-}, \tau \rightarrow \eta_{-}$and $\left.\alpha \rightarrow \alpha_{3}\right)^{8}$, we get

$$
Z_{\mathrm{CFT}} \simeq q^{-c / 24}\left(1+\frac{2 \pi i c^{-}}{\eta_{-}}\left(\frac{\sigma}{18} \frac{\alpha_{3}^{2}}{\eta_{-}^{4}}-\frac{20 \sigma^{2}}{81} \frac{\alpha_{3}^{4}}{\eta_{-}^{8}}+\cdots\right)\right)
$$

The above answer for the partition function matches precisely with the one calculated from gravity (5.25). It can also be seen easily that (5.34) reduces to the Cardy's formula for the BTZ black hole embedded in the gravitational $s l(2)$ when $\alpha_{1}=0=$ $\alpha_{2}=\alpha_{3}$.

\subsection{Generalizations to higher spin black holes in $\operatorname{shs}[\lambda]$}

One can generalize the above observations found for black holes in the $\operatorname{sl}(3 \mid 2)$ theory to the case of $\operatorname{sl}(N \mid N-1)$ theory and the Chern-Simons theory based on the superalgebra shs $[\lambda]$. The crucial observation which made it possible to obtain the partition function of the black hole from the CFT was the existence of charges by which the CFT decoupled into three bosonic sub-algebras. The charges of the black hole could then be mapped to charges in each of the sub-algebras. The black hole we studied turned out to have higher spin charge in the $s l(3)$ sub-algebra as well as a charge in the $\operatorname{sl}(2)$ part. Thus appealing to the result of [33] and the Cardy formula the entropy of the black hole was reproduced from the CFT.

Let us now examine the situation for the Chern-Simons theory based on the superalgebra shs $[\lambda]$. It is dual to the Kazama-Suzuki model based on the coset [8]

$$
\frac{S U(N+1)_{k} \times S O(2 N)_{1}}{S U(N)_{k+1} \times U(1)_{N(N+1)(k+N+1)}}
$$

The model has a $\mathcal{W}_{N+1}$ superalgebra whose global part is $\operatorname{sl}(N+1 \mid N)$. The t' Hooft limit of this coset with

$$
\lambda=\frac{N}{k+N}
$$

is dual to the shs[ $\lambda]$ Chern-Simons theory. As discussed in [43, 8] the coset admits the following decomposition

$\frac{S U(N+1)_{k} \times S O(2 N)_{1}}{S U(N)_{k+1} \times U(1)_{N(N+1)(k+N+1)}} \sim \frac{S U(k)_{N} \times S U(k)_{1}}{S U(k)_{N+1}} \times \frac{S U(N)_{k} \times S U(N)_{1}}{S U(N)_{k+1}} \times U(1)$

832 , 33 has $\sigma$ set to -1 . 
These cosets are precisely of the form considered by [2] in the minimal model/higher spin duality. Thus the bosonic part of the $\mathcal{W}_{N+1}$ superalgebra decomposes into the algebra $\mathcal{W}_{\infty}(1-\lambda) \oplus \mathcal{W}_{\infty}(\lambda) \oplus u(1)$. It's worthwhile noting that the coset is invariant under the level-rank exchange $N \longleftrightarrow k$ (or $\lambda \longleftrightarrow 1-\lambda$ ). These theories therefore have a strong-weak self-duality. Now any black hole considered in the shs $[\lambda]$ theory will carry a specific set of bosonic charges. The relation (5.37) implies that, there exists a re-definition of the charges such that they correspond to charges in the decoupled bosonic sub-algebra of the superalgebra. Note that the decomposition in (5.37) is a property of these $\mathcal{N}=2$ supersymmetric minimal models, the structure of the super-algebra is important for this decomposition just as we have seen in our study of the $\mathcal{N}=2$ super $\mathcal{W}_{3}$ algebra. The partition function of black holes carrying a specific set of higher spin charges constructed by [32] in the $\mathcal{W}_{\infty}(\lambda)$ theory has been reproduced in the CFT by the computation done in [33]. Therefore we conclude that the partition function of higher spin black holes constructed by [32] embedded in $\mathcal{W}_{\infty}(1-\lambda) \oplus \mathcal{W}_{\infty}(\lambda)$ algebra will be reproduced in the CFT. It will be interesting to find the re-definitions of the charges of the black hole so that they can be mapped to charges in the decoupled algebras for the shs $[\lambda]$ just as we have done for the $s l(3 \mid 2)$ theory. To find these redefinitions one will have to investigate the complete OPE's of the theory which is constrained by supersymmetry.

\section{Conclusions}

In this paper we have obtained the relations between the definitions of the charges and the chemical potentials between the boundary CFT and the bulk sl(3|2) ChernSimons theory. These relations are summarized in Table 1. From these relations we observed that a natural linear combinations of the bulk charges can be identified with the charges of the decoupled bosonic sub-algebras of the super- $\mathcal{W}_{3}$ CFT. We then constructed a higher spin black hole in this theory and evaluated both its entropy and partition function. The entropy was shown to be a sum of contributions from the decoupled sub-algebras. The decomposition of the CFT into decoupled sectors also enabled the evaluation of the partition function which was shown to be in precise agreement with that evaluated in the bulk.

Let us now summarize where supersymmetry plays a role in our analysis. Firstly the question of studying the effect of the $U(1)$ charge in Vasiliev theories is naturally realizable in theories based on supergroup $\operatorname{sl}(N \mid N-1)$ which contains a $U(1)$ as part of the R-symmetry. Secondly the shifts in the definition of the charges and the chemical potentials given in Table 1 was obtained from the detailed analysis the $\mathcal{N}=2$ super $\mathcal{W}_{3}$-algebra. Finally the fact that this superalgebra decouples as in (2.11) and the redefinitions of Table 1 were crucial in the simplification of the CFT partition function which is summarized in the identity (5.29). This identity related the CFT chemical potentials to that of the bulk which enabled the evaluation and 
the comparison of the partition functions evaluated in the boundary CFT and that from the bulk.

As we have discussed these observations can be generalized to the case of ChernSimons theory based on the supergroup shs $[\lambda]$. It will be interesting to find the analog of the relations given in Table 1 for this case. These relations will facilitate the evaluation the entropy of the higher spin black hole in these theories. Another direction to explore is the implication of the relations in Table 1 and its counterparts for smooth conical defects in these theories. It is possible that these classical solutions can be related to the vacuum.

\section{Acknowledgements}

We would like to thank Michael Ferlaino, Matthias Gaberdiel, Rajesh Gopakumar, S. Prem Kumar and Amitabh Virmani for useful discussions. S.D. thanks ETH, Zurich and ICTP, Trieste for hospitality during which a part of this work was completed. The work J.R.D. is partially supported by the Ramanujan fellowship DSTSR/S2/RJN-59/2009.

\section{A. OPEs of $\mathcal{N}=2$ super- $\mathcal{W}_{3}$}

Following [28], we shall list the operator product expansions which were used to calculate the Ward identities in Section 2.

$$
\begin{gathered}
J(z) J(w) \sim \frac{c / 3}{(z-y)^{2}} \\
J(z) W(w) \sim \frac{2}{(z-w)^{2}} V(w) \\
T(z) J(w) \sim \frac{J(w)}{(z-w)^{2}}+\frac{\partial_{w} J(w)}{(z-y)} \\
T(z) V(w) \sim \frac{2 V(w)}{(z-w)^{2}}+\frac{\partial_{w} V(w)}{(z-y)} \\
T(z) W(w) \sim \frac{3 W(w)}{(z-w)^{2}}+\frac{\partial_{w} W(w)}{(z-y)}
\end{gathered}
$$




$$
\begin{aligned}
V(z) V(w) \sim & \frac{2 A^{[2]}}{(z-w)^{2}}+\frac{\partial_{w} A^{[2]}}{(z-w)}+\frac{c / 2}{(z-w)^{4}} \\
V(z) W(w) \sim & \frac{1}{(z-w)} C^{[4]}+\left(\frac{3}{(z-w)^{2}}+\frac{1}{(z-w)} \partial_{w}\right) C^{[3]} \\
& +\left(\frac{20}{(z-w)^{3}}+\frac{5}{(z-w)^{2}} \partial_{w}+\frac{1}{(z-w)} \partial_{w}^{2}\right) C^{[2]}+\frac{6}{(z-w)^{4}} C^{[1]} \\
W(z) J(w) \sim & \frac{2}{(z-w)^{2}} V(w)+\frac{2}{(z-w)} \partial_{w} V(w) \\
W(z) V(w) \sim & \frac{1}{(z-w)^{3}}\left(-20 C^{[2]}+6 \partial_{w} C^{[1]}\right)+\frac{1}{(z-w)^{2}}\left(3 C^{[3]}-15 \partial_{w} C^{[2]}+3 \partial_{w}^{2} C^{[1]}\right) \\
& +\frac{1}{(z-w)}\left(-C^{[4]}+2 \partial_{w} C^{[3]}-6 \partial_{w}^{2} C^{[2]}+\partial_{w}^{3} C^{[1]}\right)+\frac{6}{(z-w)^{4}} C^{[1]} \\
W(z) W(w) \sim & \frac{c c_{W}}{3(z-w)^{6}}+\left(\frac{2}{(z-w)^{2}}+\frac{1}{(z-w)} \partial_{w}\right) B^{[4]} \\
& +\left(\frac{60}{(z-w)^{4}}+\frac{30}{(z-w)^{3}} \partial_{w}+\frac{9}{(z-w)^{2}} \partial_{w}^{2}+\frac{2}{(z-w)} \partial_{w}^{2}\right) B^{[2]} \quad(\mathrm{A} .9)
\end{aligned}
$$

The exact expressions of $A^{[]}, B^{[]}$and $C^{[]}$appearing in the OPEs above are given in 28]. However, we are interested in the semiclassical limit of $c \rightarrow \infty$. It can seen from the equations of motion that the operators $\mathcal{L}, \mathcal{V}$ and $\mathcal{W}$ scale as $O(c)$, while all the chemical potentials scale as $O(1)$. On retaining just the $O(c)$ terms in the OPEs $A^{[]}, B^{[]}$and $C^{[]}$are expressed as follows

$$
\begin{aligned}
& A^{[2]}=T-\frac{3}{2 c} J^{2}+\kappa V \\
& B^{[2]}=\frac{1}{4}\left(T+\frac{\kappa}{5} V\right)-\frac{3}{40 c} J^{2} \\
& B^{[4]}=\frac{24}{c} T^{2}-\frac{36}{c^{2}} J^{2} T+\frac{9}{2 c} J \partial^{2} J-\frac{27}{20 c} \partial^{2}\left(J^{2}\right)+\frac{24 i}{c} T V+\frac{9 i}{c} J W \\
& C^{[1]}=\frac{1}{2} J \\
& C^{[2]}=0 \\
& C^{[3]}=\frac{4}{c} J T-\frac{6}{c^{2}} J^{3}+\frac{i}{2} W+\frac{7 i}{c} J V \\
& C^{[4]}=\frac{2}{c}\left(J \partial\left(T+\kappa V+\frac{3}{2 c} J^{2}\right)-2 \partial J\left(T+\kappa V+\frac{3}{2 c} J^{2}\right)\right)
\end{aligned}
$$

While obtaining the above the fermionic operators were set to zero.

${ }^{9}$ The last term here had a 36 instead of 6 in 28] as a possible typographical error. The OPE we have written here gives the correct commutation relation. 


\section{B. Equivalence of the black hole entropy formulae}

We shall prove the equivalence of the formula for the entropy of the higher spin black hole written in terms of charges (5.8) or (5.9) with that of the formula in terms of the chemical potentials (5.26).

The formula for the entropy in terms of the chemical potentials reads as

$$
S\left(\eta_{+}, \eta_{-}, \alpha_{3}\right)=\frac{i \pi c^{+}}{6 \eta_{+}}+\frac{i \pi c^{-}}{6 \eta_{-}}\left(1+\frac{8 \sigma}{3} \frac{\alpha_{3}^{2}}{\eta_{-}^{4}}+\frac{160 \sigma^{2}}{9} \frac{\alpha_{3}^{4}}{\eta_{-}^{4}}+\cdots\right)
$$

We assume a series expansion for $S\left(\mathcal{T}_{+}, \mathcal{T}_{-}, \mathcal{W}_{-}\right)$of the form

$$
S\left(\mathcal{T}_{+}, \mathcal{T}_{-}, \mathcal{W}_{-}\right)=2 \pi \sqrt{2 \pi k_{+} \mathcal{T}_{+}}+2 \pi \sqrt{2 \pi k_{-} \mathcal{T}_{-}} \sum_{j=0}^{\infty} \chi_{j}\left(\frac{\mathcal{W}_{-}^{2}}{\mathcal{T}_{-}^{3}}\right)^{j}
$$

One can substitute $(\overline{\mathrm{B} .2})$ on the LHS of (B.1) and find $\alpha_{3}=\frac{i}{4 \pi^{2}} \frac{\partial S}{\partial \mathcal{W}}$ and $\eta_{i}=\frac{i}{4 \pi^{2}} \frac{\partial S}{\partial \mathcal{L}_{i}}$ and substitute them on the RHS of (B.1), to get the following equations

$$
\begin{aligned}
\chi_{0} & =1 \\
256 \pi \sigma \chi_{1}^{2} & =3 k \chi_{1} \\
5\left(45 k^{2} \chi_{1}^{2}+18 k^{2} \chi_{2}+524288 \pi^{2} \sigma^{2} \chi_{1}^{4}\right) & =3072 \pi k \sigma \chi_{1}\left(25 \chi_{1}^{2}+4 \chi_{2}\right)
\end{aligned}
$$

which have the solutions

$$
\chi_{0}=1, \quad \chi_{1}=\frac{3 k_{-}}{256 \pi \sigma}, \quad \chi_{2}=-\frac{105 k_{-}^{2}}{131072 \pi^{2} \sigma^{2}}, \cdots
$$

This is in precise agreement with the expansion in terms of the charges given in (5.9).

\section{References}

[1] M. Vasiliev, Nonlinear equations for symmetric massless higher spin fields in (A)dS(d), Phys.Lett. B567 (2003) 139-151, hep-th/0304049.

[2] M. R. Gaberdiel and R. Gopakumar, Minimal Model Holography, arXiv:1207.6697.

[3] M. R. Gaberdiel and R. Gopakumar, An AdS 3 Dual for Minimal Model CFTs, Phys.Rev.D 83, 066007 (2011) arXiv:1011.2986.

[4] M. Blencowe, A Consistent Interacting Massless Higher Spin Field Theory in $D=(2+1)$, Class.Quant.Grav. 6 (1989) 443.

[5] M. Henneaux and S.-J. Rey, Nonlinear $W_{\text {infinity }}$ as Asymptotic Symmetry of Three-Dimensional Higher Spin Anti-de Sitter Gravity, JHEP 1012 (2010) 007, arXiv:1008.4579. 
[6] M. Henneaux, G. Lucena Gomez, J. Park, and S.-J. Rey, Super- W(infinity) Asymptotic Symmetry of Higher-Spin AdS S Supergravity, JHEP 1206 (2012) 037, arXiv:1203.5152.

[7] A. Campoleoni, S. Fredenhagen, S. Pfenninger, and S. Theisen, Asymptotic symmetries of three-dimensional gravity coupled to higher-spin fields, JHEP 1011 (2010) 007, arXiv:1008.4744.

[8] T. Creutzig, Y. Hikida, and P. B. Ronne, Higher spin AdS $S_{3}$ supergravity and its dual CFT, JHEP 1202 (2012) 109, [arXiv:1111.2139.

[9] M. Ammon, M. Gutperle, P. Kraus, and E. Perlmutter, Black holes in three dimensional higher spin gravity: A review, arXiv:1208.5182.

[10] A. Castro, R. Gopakumar, M. Gutperle, and J. Raeymaekers, Conical Defects in Higher Spin Theories, JHEP 1202 (2012) 096, arXiv:1111.3381.

[11] A. Castro, E. Hijano, A. Lepage-Jutier, and A. Maloney, Black Holes and Singularity Resolution in Higher Spin Gravity, JHEP 1201 (2012) 031, arXiv:1110.4117.

[12] M. Ammon, M. Gutperle, P. Kraus, and E. Perlmutter, Spacetime Geometry in Higher Spin Gravity, JHEP 1110 (2011) 053, arXiv:1106.4788.

[13] H. Tan, Exploring Three-dimensional Higher-Spin Supergravity based on $\operatorname{sl}(N \mid N-1)$ Chern-Simons theories, arXiv:1208.2277.

[14] S. Datta and J. R. David, Supersymmetry of classical solutions in Chern-Simons higher spin supergravity, JHEP 1301 (2013) 146, arXiv:1208.3921.

[15] Y. Hikida, Conical defects and N=2 higher spin holography, arXiv:1212.4124.

[16] A. Schwimmer and N. Seiberg, Comments on the $N=2, N=3, N=4$ Superconformal Algebras in Two-Dimensions, Phys.Lett. B184 (1987) 191.

[17] M. Henneaux, L. Maoz, and A. Schwimmer, Asymptotic dynamics and asymptotic symmetries of three-dimensional extended AdS supergravity, Annals Phys. 282 (2000) 31-66, hep-th/9910013.

[18] J. M. Maldacena and L. Maoz, Desingularization by rotation, JHEP 0212 (2002) 055, hep-th/0012025.

[19] P. Kraus, Lectures on black holes and the AdS(3) / CFT(2) correspondence, Lect.Notes Phys. 755 (2008) 193-247, hep-th/0609074].

[20] M. Gutperle and P. Kraus, Higher Spin Black Holes, JHEP 1105 (2011) 022, arXiv:1103.4304.

[21] B. Chen, J. Long, and Y.-N. Wang, Conical Defects, Black Holes and Higher Spin (Super-)Symmetry, arXiv:1303.0109. 
[22] S. Deser, R. Jackiw, and S. Templeton, Topologically Massive Gauge Theories, Annals Phys. 140 (1982) 372-411.

[23] S. Deser and J. Kay, Topologically Massive Supergravity, Phys.Lett. B120 (1983) 97-100.

[24] E. Witten, (2+1)-Dimensional Gravity as an Exactly Soluble System, Nucl.Phys. B311 (1988) 46.

[25] A. Achucarro and P. Townsend, A Chern-Simons Action for Three-Dimensional anti-De Sitter Supergravity Theories, Phys.Lett. B180 (1986) 89.

[26] J. Izquierdo and P. Townsend, Supersymmetric space-times in (2+1) adS supergravity models, Class.Quant.Grav. 12 (1995) 895-924, gr-qc/9501018.

[27] X. Bekaert, S. Cnockaert, C. Iazeolla, and M. Vasiliev, Nonlinear higher spin theories in various dimensions, hep-th/0503128.

[28] L. Romans, The N=2 super W(3) algebra, Nucl.Phys. B369 (1992) 403-432.

[29] L. Frappat, P. Sorba, and A. Sciarrino, Dictionary on Lie superalgebras, hep-th/9607161.

[30] M. Banados, R. Canto, and S. Theisen, The Action for higher spin black holes in three dimensions, JHEP 1207 (2012) 147, arXiv:1204.5105.

[31] P. Kraus and T. Ugajin, An Entropy Formula for Higher Spin Black Holes via Conical Singularities, arXiv:1302.1583.

[32] P. Kraus and E. Perlmutter, Partition functions of higher spin black holes and their CFT duals, JHEP 1111 (2011) 061, arXiv:1108.2567].

[33] M. R. Gaberdiel, T. Hartman, and K. Jin, Higher Spin Black Holes from CFT, JHEP 1204 (2012) 103, arXiv:1203.0015.

[34] C. Peng, Dualities from higher-spin supergravity, arXiv:1211.6748.

[35] A. Perez, D. Tempo, and R. Troncoso, Higher spin gravity in 3D: black holes, global charges and thermodynamics, arXiv:1207.2844.

[36] A. Campoleoni, S. Fredenhagen, S. Pfenninger, and S. Theisen, Towards metric-like higher-spin gauge theories in three dimensions, arXiv:1208.1851.

[37] J. R. David, M. Ferlaino, and S. P. Kumar, Thermodynamics of higher spin black holes in 3D, JHEP 1211 (2012) 135, arXiv:1210.0284.

[38] B. Chen, J. Long, and Y.-N. Wang, Phase Structure of Higher Spin Black Hole, arXiv: 1212.6593 .

[39] A. Perez, D. Tempo, and R. Troncoso, Higher spin black hole entropy in three dimensions, arXiv:1301.0847. 
[40] J. de Boer and J. I. Jottar, Thermodynamics of Higher Spin Black Holes in $A d S_{3}$, arXiv:1302.0816.

[41] H. Moradi and K. Zoubos, Three-Point Functions in N=2 Higher-Spin Holography, JHEP 1304 (2013) 018, arXiv:1211.2239.

[42] T. Creutzig, Y. Hikida and P. B. Ronne, Three point functions in higher spin AdS 3 supergravity, JHEP 1301 (2013) 171, arXiv:1211.2237.

[43] R. Blumenhagen and A. Wisskirchen, Extension of the $N=2$ Virasoro algebra by two primary fields of dimension 2 and 3, Phys.Lett. B343 (1995) 168 [hep-th/9408082]. 